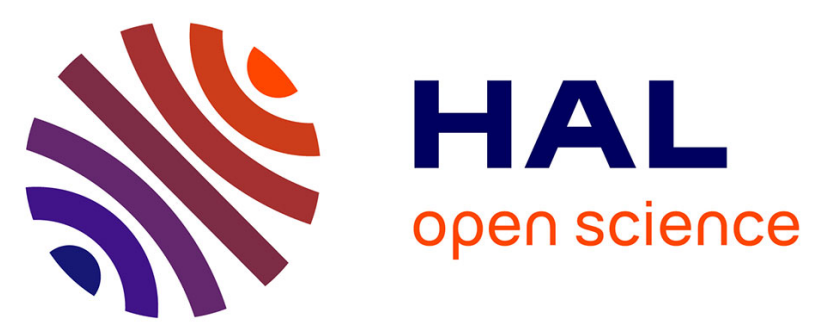

\title{
Transient non-linear dynamic analysis of automotive disc brake squeal - On the need to consider both stability and non-linear analysis \\ Jean-Jacques Sinou
}

\section{- To cite this version:}

Jean-Jacques Sinou. Transient non-linear dynamic analysis of automotive disc brake squeal - On the need to consider both stability and non-linear analysis. Mechanics Research Communications, 2010, 37, pp.96-105. 10.1016/j.mechrescom.2009.09.002 . hal-00454168

\section{HAL Id: hal-00454168 \\ https://hal.science/hal-00454168}

Submitted on 25 Sep 2012

HAL is a multi-disciplinary open access archive for the deposit and dissemination of scientific research documents, whether they are published or not. The documents may come from teaching and research institutions in France or abroad, or from public or private research centers.
L'archive ouverte pluridisciplinaire HAL, est destinée au dépôt et à la diffusion de documents scientifiques de niveau recherche, publiés ou non, émanant des établissements d'enseignement et de recherche français ou étrangers, des laboratoires publics ou privés. 


\title{
Transient non-linear dynamic analysis of automotive disc brake squeal - On the need to consider both stability and non-linear analysis
}

\author{
Jean-Jacques Sinou \\ Laboratoire de Tribologie et Dynamique des Systèmes UMR-CNRS 5513, Ecole Centrale de Lyon, 36 avenue \\ Guy de Collongue, 69134 Ecully Cedex, France \\ email: jean-jacques.sinou@ec-lyon.fr
}

\begin{abstract}
This paper outlines the non-linear transient and stationary dynamics due to friction induced vibrations in a disc brake model. Using a finite element model and the Continuous Wavelet Transform, the contributions of fundamental frequencies and harmonic components in non-linear transient and stationary dynamics are investigated for disc brake system subjected to single and multi instabilities. Results from these non-linear analyses demonstrate the complexity of the contributions of different harmonic components in transient friction-induced vibrations with the coexistence of multi-unstable modes. One of the most important contributions of this study is to illustrate the limitation of stability analysis related to transient and stationary non-linear behaviors. Stability analysis around an equilibrium point can only be used as the first step in providing information on the onset and increase of self-excited disc brake vibrations. Consequently, a complete non-linear analysis is necessary to fully predict non-linear vibration and the contribution of unstable modes. This study shows that an under-estimation of the unstable modes observed in the nonlinear time simulation can be calculated by the stability analysis. During transient vibrations, an additional unstable mode can appear. This instability is not predicted by the complex eigenvalues analysis due to the fact that linear conditions (i.e. the linearised stability around an initial equilibrium point) are not valid during transient and stationary oscillations. So new fundamental frequencies (linked to the appearance of the new unstable mode) can emerge in the signals due to the nonlinear contact and loss of contact interactions at the frictional interface. Therefore non-linear transient and stationary self-excited vibrations can become very complex and include more unstable modes than those predicted by a linearized stability analysis around a non-linear equilibrium point.
\end{abstract}

\section{Introduction}

The detection of disc brake squeal instabilities and the prediction of amplitudes during squeal events are complex tasks that have been studied for many years and continue to be a major concern in the automotive industry $[1,2]$. Nowadays, finite element models are classically used to perform two kinds of analysis for disc brake squeal: eigenvalue analysis to detect squeal frequencies and time analysis to determine self-excited vibrations during the squeal event. One of the greatest advantages of a brake finite element model is that the different parts of the brake system are modeled realistically. Therefore complex parametric studies based on an eigenvalue analysis can be extensively investigated to detect brake squeal in relation to different physical parameters [3,4]. For example, Massi et al. [5] proposed performing both stability analysis to detect system instabilities and non-linear analysis during brake simulations to reproduce squeal phenomena in the time domain. They demonstrated that the numerical and experimental results obtained are in good agreement. Chen and Zhou [6] provided time-frequency analysis of experimental results and concluded that friction vibration system is generally a linear system in the phase of vibration initiation and then becomes a nonlinear system in the phases of vibration being bounded and disappearance. They also indicated that generation of friction-induced vibration nonlinearity is attributed to the friction contact change at the interface. Lorang et al. [7] provided a theoretical discussion on the prediction of the brake squeal phenomenon based on a finite element analysis. They performed comparisons between numerical results based on the prediction of squeal frequencies (i.e. 
eigenvalue analysis), and experimental tests on squeal frequencies (based on the frequency response functions of the brake disc). The authors concluded that the numerical and experimental analyses are in good agreement regarding frequency instabilities. However, they explained that the squeal phenomenon is not yet completely understood and that it is necessary to obtain the complete non-linear dynamic responses of the squeal event. However, only few studies based on finite element models consider the transient non-linear behaviors of brake systems subjected to multi-instabilities or propose the detection of different harmonic contributions during the squeal event. As a consequence, it is difficult to track the evolutions of the fundamental frequencies and harmonic components during transient and stationary self-excited vibrations in order to better understand the mode coupling phenomenon and the coexistence of multi instabilities in non-linear transient signals.

Therefore this paper focuses on non-linear transient vibrations in brake systems by considering the Continuous Wavelet Transform. The main contribution of the present study is to explore not only the evolutions of transient non-linear quasi-periodic vibrations with multi-instabilities but also to illustrate the limitations of a local stability analysis around a given equilibrium point. The possible emergence of a additional unstable mode under transient and stationary quasi-periodic vibrations will be demonstrated even in the case where this instability and its associated unstable mode have not been previously predicted by the stability analysis of the initial equilibrium point.

Firstly, the brake system under study and the brief basic theory of the wavelet analysis with the Continuous Wavelet Transform are presented. Secondly, the stability analysis of an equilibrium point for the brake system is given. Then, parametric studies are used to investigate the different contributions of the multi-harmonic components during transient vibrations (versus the evolution of the friction coefficient). Three cases are highlighted and studied in-depth: firstly, the classical single instability phenomenon, followed by the coupling patterns that involve multi instabilities and, lastly, the emergence of a new instability not previously detected by the stability analysis around a non-linear equilibrium point. The latter case will illustrate the limitations of stability analysis (i.e. an under-prediction of the unstable modes) relating to transient and stationary non-linear self-excited vibrations.

\section{Finite element model of the brake system}

The brake system considered in this paper represents a simplified brake consisting of a disc and a pad, as illustrated in Figures 1(a-b). Hydraulic pressure is directly applied to the backplate of the pad. The friction interface is modeled by introducing contact elements between the disc and pad. Firstly, contact and loss of contact configurations at the friction interface are taken into account so that the pad and disc can separate at several local nodes during vibration. Moreover, a formulation of cubic contact force at the friction interface between the disc and the pad has been chosen to approximate the first and the third order of pad compression curves obtained from experimental tests, as shown in Figure 1(c). The friction coefficient $\mu$ is assumed to be constant for the sake of simplicity and the classical Coulomb law is applied. Therefore the non-linear contact force vectors at the friction interface along the normal direction are defined by

$$
\begin{gathered}
F_{\text {contact }, X}^{d}=k_{l}\left(x_{d}-x_{p}\right)+k_{n l}\left(x_{d}-x_{p}\right)^{3} \quad \text { if }\left(x_{p}-x_{d}\right)>0 \quad(0 \text { otherwise }) \\
F_{\text {contact }, X}^{p}=-F_{\text {contact }, X}^{d}
\end{gathered}
$$

where $d$ and $p$ define the disc and the pad, respectively. $k_{l}$ and $k_{n l}$ correspond to the linear and non-linear stiffnesses at the friction interface between the disc and the pad. $x_{d}$ and $x_{p}$ are the displacements of the disc and pad, respectively, in the direction normal to the contact surface. Then the non-linear contact force vectors at the friction interface along the tangential direction are defined by $F_{\text {contact, } Y}^{p}=\mu F_{\text {contact, } X}^{p} \operatorname{sign}\left(v_{r}\right)$ and $F_{\text {contact }, Y}^{d}=\mu F_{\text {contact }, X}^{d} \operatorname{sign}\left(v_{r}\right)$ where $v_{r}$ is the relative velocity between the pad and disc. As previously 


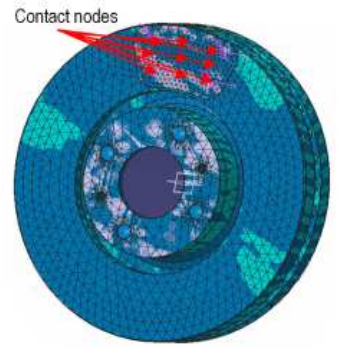

(a) Disc

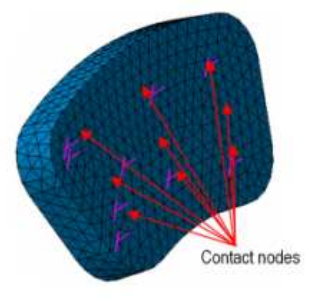

(b) Pad

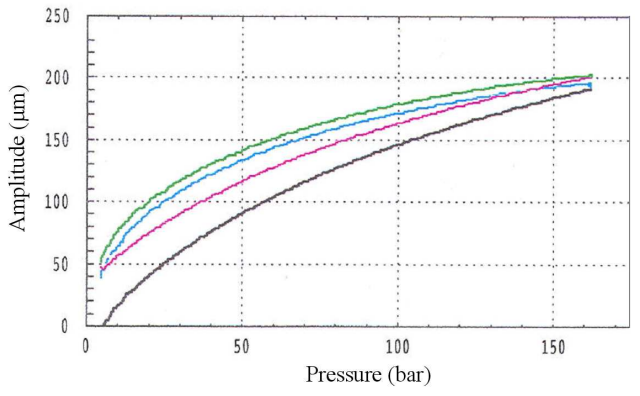

(c) Experimental pad compression

Figure 1: Finite element of the brake system ((a) Disc and (b) Pad) and experimental tests ((c) Pad compression)

explained by Kang et al. [8,9], the radial component of friction force can change the formation of the limit cycle if an equilibrium point of the brake system becomes unstable. However, this contribution has been neglected in this study for the sake of simplicity. For more details about the effect of additional radial components of friction force, those interested can refer to [10].

Finally, the brake system (disc and pad) is reduced by using a Craig and Bampton technique, keeping the contact nodes at the disc/pad interface and retaining the first fifty eigenmodes of each component of the brake system [11]. The equation of motion for the brake system is

$$
\mathbf{M} \ddot{\mathbf{x}}+\mathbf{C} \dot{\mathbf{x}}+\mathbf{K x}=\mathbf{F}_{\mathrm{NL}}+\mathbf{F}_{\mathrm{ext}}
$$

where $\mathbf{M}, \mathbf{C}$ and $\mathbf{K}$ are mass, damping and stiffness matrices, respectively, and $\mathbf{x}$ is the generalized displacement vector while the dot denotes derivative with respect to time. $\mathbf{F}_{\mathbf{N L}}$ contains the linear and non-linear contact forces at the frictional interface. It can be noted that the nonlinearities at the friction interfaces are both the cubic nonlinear terms and the possible loss of contact between nodes on the disc surface and nodes on the pad surface. $\mathbf{F}_{\text {ext }}$ is the vector force due to brake pressure applied on the pad.

\section{Continuous Wavelet Transform}

It is well known that the conventional fast Fourier transform (FFT)-based spectral analysis method is suitable for analyzing steady-state vibration signals, but provides poor representation of signals well localized in time. Consequently, time-scale signal processing tools have to be used to provide a good description of non-linear contributions during the non-stationary transient signal of a brake system. In 1983, Morlet [12] proposed applying the wavelet approach to analyze the vibration of systems. Newland [13] proposed applying the Continuous Wavelet Transform (CWT) to obtain the characteristics of transient responses and changes in the properties of non-stationary signals of mechanical structures. A theoretical background can be found in [14-16] for readers interested in the subject.

The wavelet analysis transforms a signal into wavelets that are well localised both in frequency and time. The Continuous Wavelet Transform (CWT) of a function $f(t)$ is a wavelet transform defined by

$$
W(a, b)=\int_{-\infty}^{+\infty} f(t) \psi_{a, b}^{*}(t) d t \quad \text { where } \quad \psi_{a, b}(t)=\frac{1}{\sqrt{a}} \psi\left(\frac{t-b}{a}\right)
$$

$\psi_{a, b}(t)$ are the daughter wavelets (i.e. the dilated and shifted versions of the "'mother" wavelet $\psi$ that is continuous in both time and frequency). $a$ defines the scale parameter, and $b$ corresponds to the time parameter. 
The asterisk $\psi_{a, b}^{*}$ indicates the complex conjugate of $\psi_{a, b}$. The following admissibility condition has to be satisfied $0<C_{\psi}<+\infty$ where $C_{\psi}$ defines the admissibility constant $C_{\psi}=\int_{-\infty}^{+\infty} \frac{|\hat{\psi}(\omega)|^{2}}{|\omega|} d \omega$ and $\hat{\psi}$ is the Fourier transform of $\psi$. It can be given by $\hat{\psi}=\int_{-\infty}^{+\infty} f(t) e^{i \omega t} d t$. For a time signal $f(t)$ represented by $N$ sampled data points (with uniform time step $\delta t$ ), the Continuous Wavelet Transform of equation (4) is a convolution of the data sequence $f\left(n^{\prime}\right)$ (with $n^{\prime}=1, \ldots, N$ ) with a scaled and normalized wavelet. It can be represented as follows:

$$
C(a, n)=\sum_{n^{\prime}=0}^{N-1} f\left(n^{\prime}\right) \sqrt{\frac{\delta t}{a}} \Psi_{0}^{*}\left(\frac{\left(n^{\prime}-n\right) \delta t}{a}\right)
$$

where $n$ defines the localized time index and $\delta t$ is the sampling interval. One of the most important points is the specification of an appropriate type of the mother wavelet that serves as the source function from which scaled and translated basis functions are constructed. In this paper, the Morlet mother wavelet has been chosen due to the fact that it is one of the most commonly used CWT wavelets (quite well localized in both time and frequency space). It is defined as the following in the time domain: $\psi_{0}(\eta)=\pi^{-\frac{1}{4}} e^{i m \eta} e^{-\frac{\eta^{2}}{2}}$ where $m$ is the wavenumber and $\eta$ is a non-dimensional time parameter. The wavelet function contains unit energy at every scale due to the normalization of the mother wavelet. The wavelet power is then defined as $|C(a, n)|^{2}$.

For this study, part of the Continuous Wavelet Transform software includes code originally written by C. Torrence and G. Compo [17].

\section{Numerical study}

\subsection{Instability of the equilibrium point}

The non-linear oscillations of the brake system are due to the frictional interface that generates self-excited vibrations: the friction-induced vibrations lead to the divergence of an equilibrium point of the non-linear brake system, called "'system instability". Therefore the first step is to estimate the stability of the equilibrium points for a given set of parameters [18]. Considering the previous non-linear system (3), stability is calculated by considering the linearized system at the equilibrium point $\mathbf{x}_{\mathbf{0}}$ (i.e. $\mathbf{K} \mathbf{x}_{\mathbf{0}}=\mathbf{F}_{\mathrm{NL}}+\mathbf{F}_{\text {ext }}$ ). The linearized system can be written in the following way

$$
\mathbf{M} \ddot{\overline{\mathbf{x}}}+\mathbf{C} \dot{\overline{\mathbf{x}}}+\left(\mathbf{K}-\mathbf{K}_{\mathbf{L}}\left(\mathbf{x}_{\mathbf{0}}\right)\right) \overline{\mathbf{x}}=\mathbf{0}
$$

where $\overline{\mathbf{x}}$ defines the perturbation around the equilibrium point (i.e. $\mathbf{x}=\mathbf{x}_{\mathbf{0}}+\overline{\mathbf{x}}$ ), and $\mathbf{K}_{\mathbf{L}}$ is the linearized expression of the non-linear frictional contact (i.e. $\left.\left.\mathbf{F}_{\mathbf{N L}}(\overline{\mathbf{x}}) \approx \sum_{i} \frac{\partial \mathbf{F}_{\mathbf{N L}}(\overline{\mathbf{x}})}{\partial \bar{x}_{i}}\right|_{\mathbf{x}_{\mathbf{0}}} \bar{x}_{i}=\mathbf{K}_{\mathbf{L}}\left(\mathbf{x}_{\mathbf{0}}\right) \overline{\mathbf{x}}\right)$. The complex eigenvalue analysis then provides the stability of the equilibrium points: if the real part of all the eigenvalues of the system (6) remains negative, the equilibrium point $\mathbf{x}_{\mathbf{0}}$ under study is stable. If at least one of the eigenvalues has a positive real part, an instability of the system is generated for the equilibrium point $\mathbf{x}_{\mathbf{0}}$ under study (i.e. this equilibrium point is unstable, leading to oscillations of the non-linear brake system). Figures 2 show the complex eigenvalue analysis as a function of the friction coefficient $\mu$. It can be seen that increasing the friction coefficient increases the number of instabilities. The first instability is detected for a friction value of $\mu=0.26$. The value of the associated unstable mode is $1510 \mathrm{~Hz}$, as indicated in Figure 2(a). The second instability occurs for a friction value higher than $\mu=0.28$. The associated unstable mode is detected at $920 \mathrm{~Hz}$.

\subsection{Transient dynamics of the brake system}

As explained previously, if an equilibrium point becomes unstable, non-linear transient and/or stationary selfexcited vibrations can be generated. In this section, transient non-linear vibrations are investigated in relation 

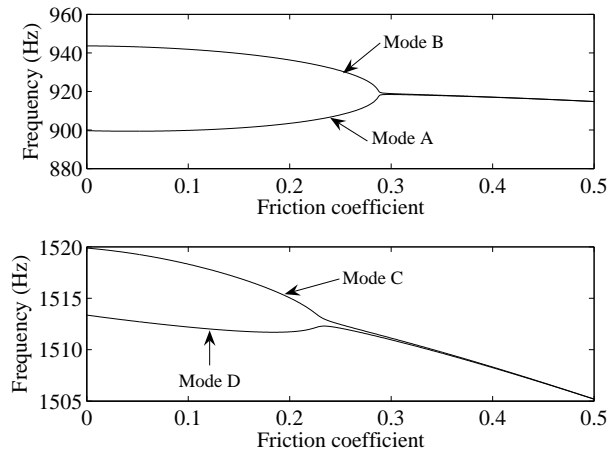

(a)

(b)

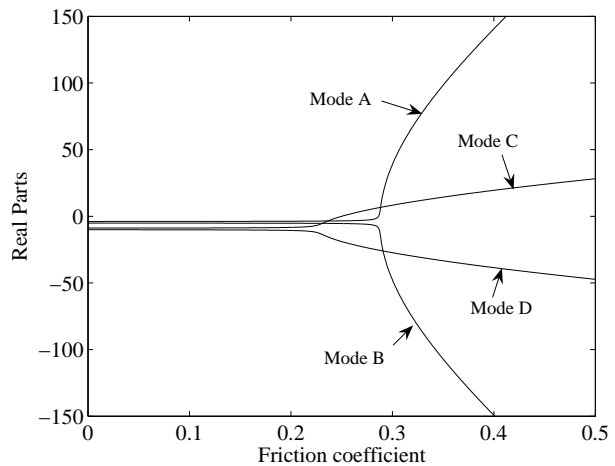

Figure 2: Stability analysis of the brake system (a) Frequencies (b) Real parts

\begin{tabular}{ccc}
\hline Friction coefficient & Frequency $f_{1}(\mathrm{~Hz})$ & Frequency $f_{2}(\mathrm{~Hz})$ \\
\hline 0.26 (case 1$)$ & & \\
0.29 & - & 1512 \\
0.3 & 898 & 1497 \\
0.35 & 897 & 1495 \\
0.26 (case 2$)$ & 904 & 1495 \\
\end{tabular}

Table 1: Fundamental frequencies of the nonlinear responses

with the contribution of the harmonic components of the fundamental unstable frequencies. This second step is essential in a design process aimed at clearly understanding the evolutions of the non-linear behavior for a brake system with an unstable equilibrium point. In this paper, the time history responses of the nonlinear brake system defined in equation (3) are solved by using the Adams-Bashforth-Moulton PECE solver.

In this section, it will be demonstrated that the transient non-linear friction-induced vibrations can be more or less complex. It will be illustrated that only considering the stability analysis around an equilibrium point is not sufficient for predicting the possible number of unstable modes (i.e. the fundamental frequency components) of the complete non-linear transient and stationary self-excited vibrations. It will be shown that an underprediction of the unstable modes (that are present in the time simulation) can be estimated by the linear analysis. In the following, the wavelet power spectrum and frequency analysis of the nonlinear transient responses are carried out to compare the dynamic behavior obtained by time simulations with the complex eigenvalues calculated in the previous section Stability analysis. We remind that the linear stability analysis and the linearization around an initial equilibrium point are not valid for the transient analysis. However, the main purpose of this "comparison" is only to characterize all the resonances peaks of the non-linear transient vibrations and to check the dynamic similarities between the linear analysis (for the detection and prediction of instabilities) and the time analysis (for the characterization of the non-linear dynamic behavior in time domain). 
(a)

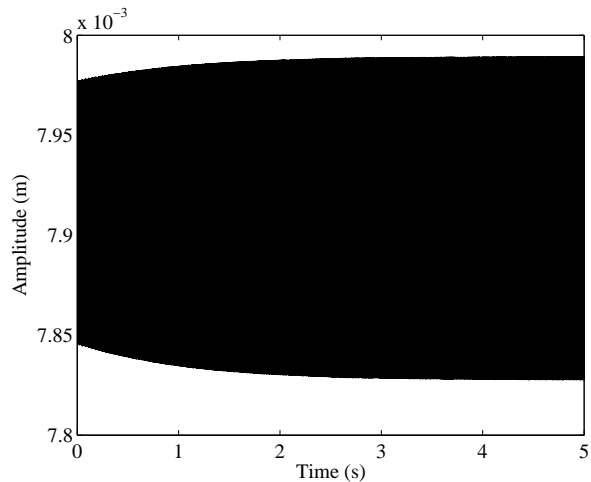

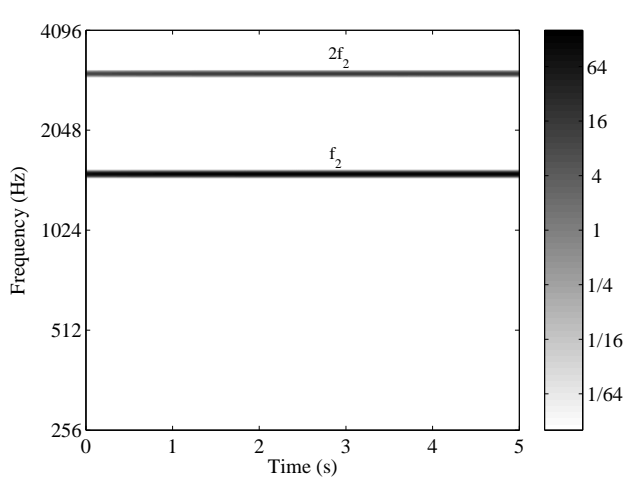

(b)

Figure 3: Transient non-linear responses of the brake system for $\mu=0.26$ (a) Time history for $t=[0 ; 5] s(\mathrm{~b})$ Wavelet power spectrum for $t=[0 ; 5] s$

\subsubsection{Case 1: the single instability}

First of all, Figure 3(a) illustrates the transient responses for one of the most simple and classical non-linear behaviors observed during a single instability phenomenon. The displacement chosen corresponds to one degreeof-freedom of the pad in the direction normal to the contact surface. Moreover, the value of the constant friction coefficient for this case is $\mu=0.26$.

It can be seen that the displacement increases until the periodic non-linear oscillations are obtained. The associated wavelet power spectrum during the transient oscillations is given in Figure 3(b). It clearly appears that the resonance peaks can be compared with the contribution of the fundamental frequency $f_{2}$ corresponding to the unstable frequency of the mode (as previously indicated in section Stability analysis) and its $2 \times$ harmonic component. The values of $f_{2}$ (for the stationary self-excited oscillations) are indicated in Table 1.

Both the $1 \times$ and $2 \times$ harmonic components are present for all the time history responses. However, it can be reminded that, theoretically, the stability analysis of the linearized system around an initial equilibrium point cannot predict the fundamental frequencies of the transient and stationary signals (i.e. the eigenvalues calculated in the previous section Stability analysis have been estimated for an equilibrium point that is not valid for the non-smooth system during transient and stationary vibrations).

Therefore this first case illustrates the fact that the transient non-linear oscillations due to an unstable equilibrium point can be very simple: this study gives an example of the transient non-linear self-excited oscillations of the brake system with the participation of a "single instability" (i.e. with only one fundamental frequency and its harmonics in the non-linear vibrations generated by a single unstable equilibrium point). In this first case, the appearances of harmonic components are only due to the non-linear stiffness: loss of contact between nodes on the disc surface and nodes on the pad surface is not observed.

\subsubsection{Case 2: the classical case of "multi-instabilities"}

As previously shown in section Stability analysis, the brake system under study can be affected by two "instabilities" if the friction coefficient is greater than $\mu=0.28$. In this part of the paper, the transient vibrations generated for different friction coefficients (with $\mu>0.28$ ) will be investigated.

First, Figures 4(a) illustrates the non-linear transient oscillations in the case of $\mu=0.29$. The associated wavelet power spectrum and the contributions of combinations of harmonics for the pad in the direction normal to the contact surfaces are given in Figure 4(b).

As indicated in Figure 4(a), the highest amplitudes are observed during the transient oscillations, just before 
the quasi-periodic oscillations of the brake system. This first observation is very important because it illustrates the fact that a brake system cannot be thoroughly validated in a design process if all the transient self-excited vibrations are not examined in detail. Even if the brake system can perform properly during the final quasiperiodic vibrations (i.e. the stationary self-excited vibrations are assumed to be small enough), the transient behavior of the brake system can be a key issue in brake development for predicting dangerous or favorable conditions.

Figure 4(b) shows the resonance peaks during the transient vibrations by using the Continuous Wavelet Transform. Even if the stability analysis is not sufficient and valid to estimate the fundamental frequencies of the non-linear system under study (i.e. a non-smooth system), it appears that the modes involved in the mechanism of friction induced vibrations have been previously " predicted" under the stability analysis. The fundamental frequency of the first instability (i.e. $f_{2}$ ) and the fundamental frequency of the second instability (i.e. $f_{1}$ ) are both present in the wavelet power spectrum of the non-linear transient signal. The values of $f_{1}$ and $f_{2}$ (for the stationary self-excited oscillations) are indicated in Table 1. In addition of the two fundamental frequencies ( $f_{1}$ and $f_{2}$ ), the harmonics $\left(n f_{i}\right.$ for $i=1,2$ and $n$ positive integer) and combination harmonics $\left( \pm n f_{1} \pm m f_{2}\right.$ with $n$ and $m$ positive integers) are indicated in Figure 4(b) by using the Continuous Wavelet Transform. This fact clearly illustrates the interactions of the two instabilities that generate sum and difference frequencies $\pm n f_{1} \pm m f_{2}$ with $n$ and $m$ positive integers. The component $-f_{1}+f_{2}$ is prominent. Moreover, the wavelet power spectrum indicates the combination harmonics $f_{1}+f_{2}, 3 f_{1}-f_{2},-6 f_{1}+4 f_{2}, 4 f_{1}-2 f_{2},-3 f_{1}+2 f_{2}$, $2 f_{1}-f_{2}$ and $7 f_{1}-4 f_{2}$. However, all these combination harmonics are less significant than fundamental components $f_{1}$ and $f_{2}$, and the combination component $-f_{1}+f_{2}$. It should be noted that the presence of these combination harmonics is indicative of "strong" coupling of the two unstable modes leading to quasi-periodic self-excited vibrations of the non-linear brake system. Finally, the second harmonic component of the first and second instabilities (i.e. $2 f_{1}$ and $2 f_{2}$ ) are also observed. As explained previously, the occurrence of harmonic components and combination harmonics is due to the non-linear stiffness and contact and loss of contact interactions at the frictional interface between disc and pad. The number of loss of contact between nodes on the disc surface and nodes on the pad surface are indicated in Figure 5.

Showing Figure 4(b), the initial increase (for $t=[0 ; 1] s$ ) of the oscillations (around the unstable equilibrium point) is harmonic Oscillations are governed by the fundamental frequency $f_{1}$ : the main harmonic coincident with the "most unstable frequency" predicted by the linear model (i.e. the unstable frequency associated with the highest real part). The fundamental frequency $f_{1}$ can be clearly distinguished in the wavelet power spectrum for $t=[0 ; 1] \mathrm{s}$. Figure 5 shows that all the nodes at the frictional interface of the disc and the pad are in contact. When the amplitudes of the brake system become more significant, oscillations become more complex with contact and loss of contact. One or two nodes at the frictional interface separate (see Figure 5 - zoom 1 for $t=[1.04,1.08] \mathrm{s})$. Occurrences of the second fundamental frequency $f_{2}$ and of the combination component $-f_{1}+f_{2}$ are detected. Finally, the stationary oscillations are still complex with contact and loss of contact between nodes on the disc surface and nodes on the pad surface (see Figure 5 - zoom 2). All the combination harmonics described previously appear. What is more, it can be seen that the transient non-linear oscillations and stationary quasi-periodic signals are always mainly governed by $f_{1}, f_{2}$ and $-f_{1}+f_{2}$.

In conclusion, the transient non-linear vibrations are not only composed of fundamental frequencies $f_{1}$ and/or $f_{2}$ but also the harmonic combinations that correspond to the coexistence of the two instabilities in the brake system. Moreover, it can be noted that the frequency components observed in the wavelet power spectrum do not indicate the presence of a new "instability" but illustrate the harmonics of fundamental frequencies $f_{1}$ and $f_{2}$ that can contribute to the overall vibration during the transient and stationary periods.

Secondly, Figures 6(a) and (b) illustrate the transient and stationary quasi-periodic vibrations for the case of $\mu=0.3$. Although the variation of the friction coefficient is not very significant (less than $4 \%$ ), the wavelet power spectrum appears to be very complex with the presence of many new harmonic combinations. The initial increases of oscillations (for $t=[0 ; 0.5] \mathrm{s}$ ) are composed by the first fundamental frequency $f_{1}$ and its second 
(a)

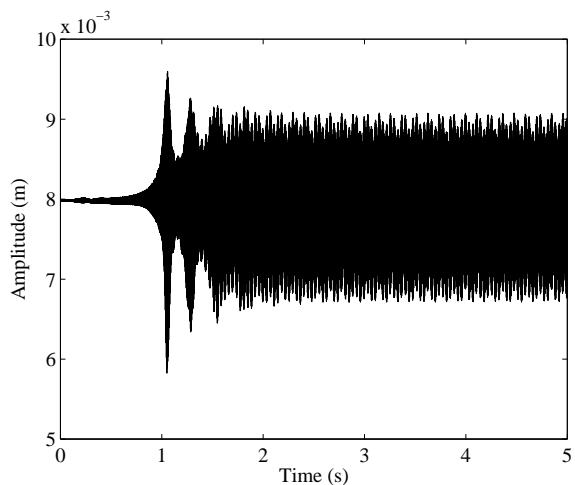

(b)

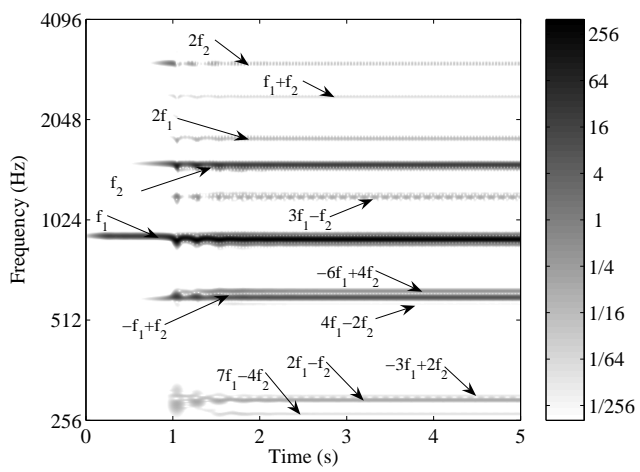

Figure 4: Transient non-linear responses of the brake system for $\mu=0.29$ (a) Time history for $t=[0 ; 5] s$ (b) Wavelet power spectrum for $t=[0 ; 5] s$

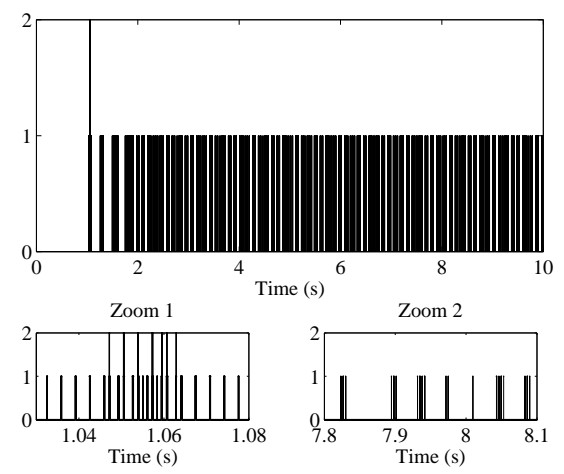

Figure 5: Number of loss of contact during the transient vibration for $\mu=0.29$

and third harmonic components (i.e. $2 f_{1}$ and $3 f_{1}$ ). This can be explained by the fact that the initial increase of the friction-induced vibrations is governed by the "most unstable" mode at the unstable equilibrium point (i.e. the mode that corresponds to the eigenvalue with the greater positive real part, as indicated in Figure 2). Then, the second fundamental frequency $f_{2}$ and interactions of the two instabilities that produce harmonic combinations $\pm n f_{1} \pm m f_{2}$ (with $n$ and $m$ positive integers) appear when the non-linear transient vibrations are maximal (at $t=0.8 \mathrm{~s}$ ). Then, all these contributions are present for the stationary quasi-periodic oscillations (that are observed for $t>2 s$ ). Even if certain harmonic combinations appear to be very close (see for example frequency ranges $[200-400] H z$ or $[500-800] H z)$, they are clearly distinguishable. Figure 6(b) shows that fundamental frequencies $f_{1}$ and $f_{2}$ and harmonic components $\left(2 f_{1}, 3 f_{1}\right.$ and $\left.2 f_{2}\right)$, as wel as the harmonic combinations (see for example $-f_{1}+f_{2},-6 f_{1}+4 f_{2},-4 f_{1}+3 f_{2}, 4 f_{1}-2 f_{2},-4 f_{1}+3 f_{2}, 2 f_{1}-f_{2},-3 f_{1}+$ $2 f_{2}$ and $7 f_{1}-4 f_{2}$ ) correspond to the most significant contributions in the complex non-linear transient and stationary quasi-periodic vibrations. Therefore these strong participations of the harmonic combinations of the two instabilities (i.e. $\pm n f_{1} \pm m f_{2}$ with $n$ and $m$ positive integers) are indicative of strong "coupling" of the two unstable modes that lead to complex transient and stationary quasi-periodic oscillations. Moreover, other combinations of less significance are also observed, as indicated in Figure 6(b). These harmonic combinations correspond to the upper orders of the sum and difference frequencies (see for example $12 f_{1}-7 f_{2},-8 f_{1}+5 f_{2}$, $-13 f_{1}+8 f_{2}, 14 f_{1}-8 f_{2}$ and $9 f_{1}+5 f_{2}$ ). The values of $f_{1}$ and $f_{2}$ (for the stationary self-excited oscillations) 
(a)

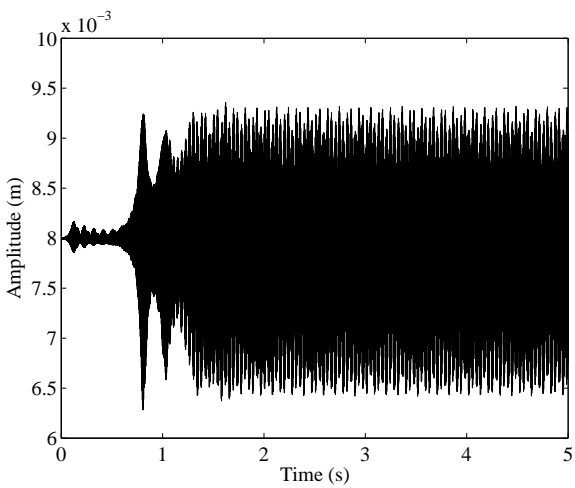

(b)

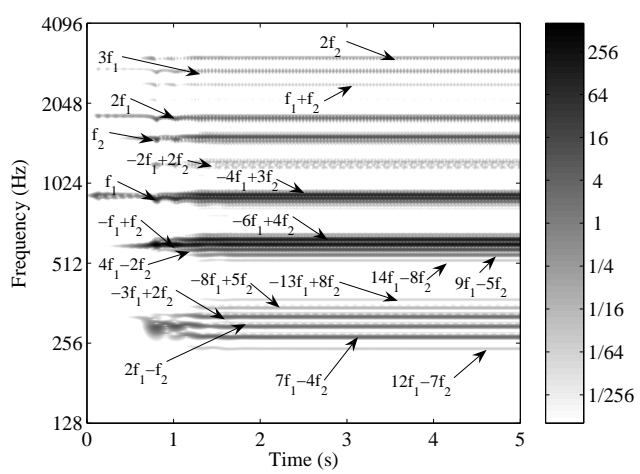

Figure 6: Transient non-linear responses of the brake system for $\mu=0.3$ (a) Time history for $t=[0 ; 5] s$ (b) Wavelet power spectrum for $t=[0 ; 5] s$

are indicated in Table 1.

Finally, Figures 7(a), (b), (c) and (d) illustrate the transient and stationary quasi-periodic vibrations for the case of $\mu=0.35$. As explained previously, the transient and stationary oscillations are composed of fundamental frequencies $f_{1}$ and $f_{2}$, harmonic components $2 f_{1}$ and $3 f_{1}$, and harmonic combinations $2 f_{1}-f_{2},-f_{1}+f_{2}$. Other combinations $\left(f_{1}+f_{2},-3 f_{1}+2 f_{2}, 4 f_{1}-2 f_{2},-2 f_{1}+2 f_{2}\right.$ and $\left.3 f_{1}-f_{2}\right)$ of less significance can also be detected. Thus all the harmonic combinations correspond to the lower orders of the sum and difference frequencies.

Two behaviors can be observed when examining the time history of the non-linear responses (see Figures 7(a) and (c)): firstly, a very fast increase of the transient oscillations for $t=[0 ; 0.1] \mathrm{s}$ followed by a decrease for $t=[0.1 ; 1]$ s. During this first part of the system's non-linear behavior, the fundamental frequency $f_{1}$ and the associated harmonic components $2 f_{1}$ and $3 f_{1}$ are predominant. These observations can be explained by the fact that the non-linear transient vibrations are first governed by the most unstable mode (i.e. the mode with the greater real part at the unstable equilibrium point). A small contribution of the combination frequencies $4 f_{1}-3 f_{2}$ and $4 f_{1}-2 f_{2}$ is observable. Then, during the second part of the transient oscillations (for $t=$ $[1 ; 5] s)$, an increase of the transient amplitudes is seen while increases of the resonances satisfy relationships $\pm n f_{1} \pm m f_{2}$ (with $\mathrm{n}$ and $\mathrm{m}$ positive integers). These interactions between the two unstable mode combination frequencies are clearly identified by considering harmonic combinations $-f_{1}+f_{2}$ and $2 f_{1}-f_{2}$. As indicated in Figure 7(d), harmonic combination $-f_{1}+f_{2}$ is prominent, being equal to $f_{1}$. Then, for $t>2 s$, the fundamental frequency $f_{2}$ and other combinations of less significance appear (see for example $3 f_{1}-f_{2},-2 f_{1}+2 f_{2}, 4 f_{1}-2 f_{2}$ and $f_{1}+f_{2}$ ). Moreover, the stationary quasi-periodic amplitudes of the brake system is composed of the two fundamental frequencies $f_{1}$ and $f_{2}$, the harmonic components $n f_{i}$ (for $i=1,2$ and $n$ positive integer) and combinations $\pm n f_{1} \pm m f_{2}$ (with $n$ and $m$ positive integers). Finally, harmonic combinations $4 f_{1}-3 f_{2}$ and $4 f_{1}-2 f_{2}$, which are present in the first part of the transient vibrations (for $t=[0: 1] s$ ), disappear in the second part of the transient oscillations for $t>1 s$. Therefore it can be concluded that the non-linear transient vibrations are complex, with increasing or decreasing harmonic combinations that illustrate the coexistence and strong interaction of the two unstable modes of fundamental frequencies $f_{1}$ and $f_{2}$, respectively. The values of $f_{1}$ and $f_{2}$ (for the stationary self-excited oscillations) are indicated in Table 1 . In comparison with the two previous cases (for $\mu=0.29$ and $\mu=0.3$ ), the maximum transient non-linear amplitudes are obtained more rapidly for $t=0.05 \mathrm{~s}$, as illustrated in Figure 7(a). Thus in this section Case 2: the classical case of " multiinstabilities"', it is shown that transient and stationary non-linear behaviors can be composed by not only the fundamental frequencies of the unstable modes but also the associated harmonic components and associated 
(a)

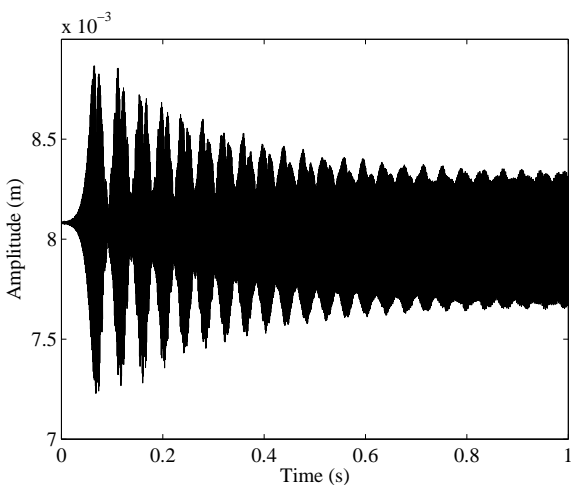

(c)

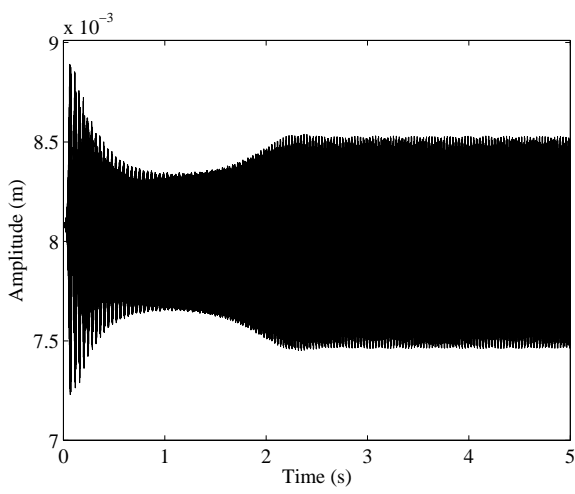

(b)

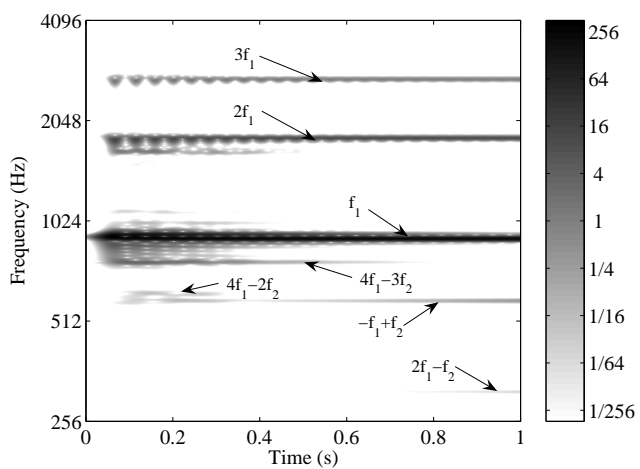

(d)

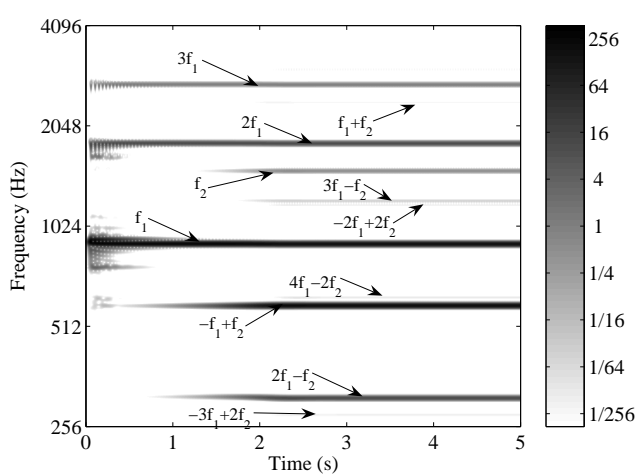

Figure 7: Transient non-linear responses of the brake system for $\mu=0.35$ (a) Time history for $t=[0 ; 1] \mathrm{s}$ (b) Wavelet power spectrum for $t=[0 ; 1] s$ (c) Time history for $t=[0 ; 5] s$ (d) Wavelet power spectrum for $t=[0 ; 5] s$

combination of frequency components. It is also shown that the harmonic components and combinations of frequencies (that correspond to "strong coupling" or the interaction of the two unstable modes) cannot be neglected when attempting to avoid defective brake system design.

For the last two cases ( $\mu=0.3$ and $\mu=0.35$ ), the number of loss of contact is indicated in Figures 8(a) and (b). For $\mu=0.3$, the phenomenon of contact and loss of contact is observed during the transient and stationary vibrations. Due to the "complex" non-linear behavior generated by this non-smooth nonlinearity, strong participations of the harmonic combinations of the two instabilities (i.e. $\pm n f_{1} \pm m f_{2}$ with $n$ and $m$ positive integers) are present, as previously explained (see the previous paragraph and Figure Figures 6(d)). For $\mu=$ 0.35 , loss of contact between nodes on the disc surface and nodes on the pad surface is only observed during the first part of the system's non-linear behavior (previously defined in Figure 7(a) and (b)). So, the stationary vibrations appear to be "less complex" (i.e. the number of harmonics combinations is less important).

In conclusion, the nonlinear transient and stationary vibrations for the last two cases $(\mu=0.3$ and $\mu=0.35$ ) can be complex due to the presence of not only the fundamental "unstable" frequencies, but also their harmonic components and the combination of frequency components. The main resonances for the initial growth are coincident with the two unstable modes predicted by the linearized stability analysis around the initial equilibrium point. However, we remind that the stability analysis can not be used to predict the resonance peaks during the transient and stationary non-linear vibrations. 
(a)
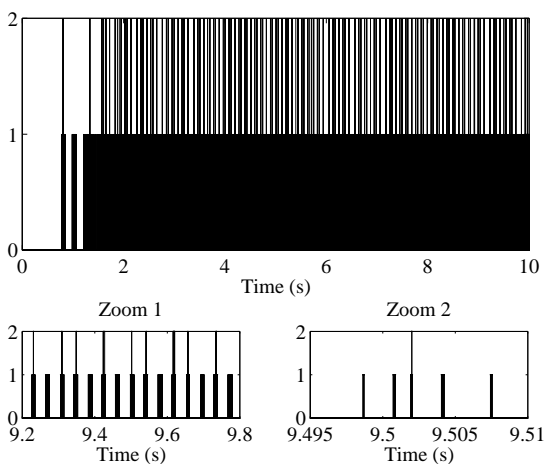

(b)
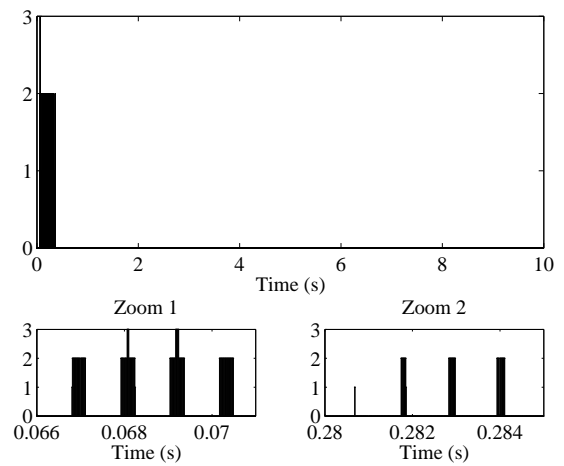

Figure 8: Number of loss of contact during the transient vibration for (a) $\mu=0.3$ and (b) $\mu=0.35$

\subsubsection{Case 3: limitation of the local stability analysis of the equilibrium point}

This aim of this section is to illustrate that the stability analysis (presented in section Instability of the equilibrium point) cannot be used as a robust indicator of brake system design. More particularly, it will be shown that an eigenvalue analysis around an equilibrium point cannot prevent all the unstable frequencies in the non-linear transient and stationary responses for a given friction coefficient and so cannot be used for detecting all the fundamental frequencies and multi-harmonic components.

To illustrate this fact, the non-linear transient vibrations of the system are investigated for $\mu=0.26$, as previously done in section Case 1: single instability. However, in this part of the section, the initial perturbations introduced in the non-linear system are bigger than those of the first case. This new initial condition is chosen as being "far" or very different from the initial non-linear equilibrium point, by keeping "'local instability" (defined by the fundamental frequency $f_{2}$ ).

To aid the reader's understanding, it should be noted that only one unstable mode (with the fundamental frequency $f_{2}$ ) has been detected previously by eigenvalue analysis around the non-linear equilibrium point (defined in section Instability of the equilibrium point).

Therefore Figures 9(a) and (b) give the transient and stationary quasi-periodic vibrations for the case of $\mu=$ 0.26 , by considering this new disturbance of the equilibrium point. By taking into account the time history of the non-linear responses (see Figure 9(a)), two dynamic behaviors for the brake system are observed: firstly, a "simple" increase of the transient oscillations for $t=[0 ; 7] \mathrm{s}$. Secondly, a "complex" non-linear transient behavior for $t=[7 ; 11] s$ until the stationary amplitudes are reached for $t=11 \mathrm{~s}$. As indicated in Figure 9(b), only the fundamental frequency $f_{2}$ and its harmonic components $2 f_{2}$ are present during the first part of the non-linear behavior of the system (for $t=[0 ; 7] s$ ).

As explained previously, the stability analysis performed in section Instability of the equilibrium point is investigated by determining the eigenvalues of the linearized equations (6) around the equilibrium points obtained by solving the static non-linear equations. Thus this initial rate of increase of non-linear amplitudes is in agreement with the eigenvalue analysis performed previously in section Instability of the equilibrium point. The increasing non-linear transient amplitudes are governed only by the fundamental frequency $f_{2}$ (corresponding to the unstable mode) and its second harmonic component $2 f_{2}$. Therefore it should be recalled that even if the initial disturbances were chosen as being "far" or different from those chosen for the first case Case 1: the single instability, these initial disturbances do not affect the eigenvalue analysis (i.e. we are at the same local non-linear equilibrium point with a stability analysis that indicates the occurrence of an instability governed by unstable mode $f_{2}$. This is why the onset of the non-linear transient vibration is in agreement with the first case Case 1: single instability: the initial oscillation starts under linear conditions with the main resonance coinci- 
dent with the unstable frequency $f_{2}$, and then the emergence of its second harmonic component $2 f_{2}$. However, for the second part of the transient and stationary non-linear behavior (for $t=[7 ; 12] s$ ), the non-linear signal appear to be more complex is composed by the fundamental frequency $f_{2}$ and its harmonics, as well as the fundamental frequency $f_{1}$ with its harmonics and harmonic combination $\pm n f_{1} \pm m f_{2}$ (with $n$ and $m$ positive integers). The values of $f_{1}$ and $f_{2}$ (for the stationary self-excited oscillations) are indicated in Table 1.

It clearly appears that all the transient and stationary non-linear oscillations can become more complex and be governed by both the initial unstable mode and by the contributions of the nonlinearities that may lead to new instabilities in the brake system. Therefore the previous local stability of the equilibrium point cannot be considered during the transient vibrations of the non-linear system. Moreover, it is shown that the new fundamental frequency $f_{1}$ corresponds to the unstable mode previously obtained for $\mu>0.28$. Now, the fundamental frequency $f_{1}$ and the combination harmonics $-f_{1}+f_{2}$ are predominant in the transient and stationary signal. The contribution of the fundamental frequency $f_{2}$ appears to be less significant. Moreover, other contributions such as $2 f_{1}, 3 f_{1}, 2 f_{1}-f_{2},-3 f_{1}+2 f_{2},-2 f_{1}+2 f_{2},-f_{1}+2 f_{2}$ and $f_{1}+f_{2}$ are now detectable. It may be concluded that the non-linear transient behavior of the brake system has changed drastically. Thus in the case under study, the transient and stationary amplitudes are composed by both the two fundamental frequencies $f_{1}$ and $f_{2}$, harmonic components $n f_{i}$ (for $i=1,2$ and $n$ positive integer) and harmonic combinations $\pm n f_{1} \pm m f_{2}$ (with $n$ and $m$ positive integers), despite the fact that the unstable mode associated with the frequency $f_{1}$ was not predicted previously by the stability analysis for the given friction coefficient $\mu=0.26$.

Finally, Figures 11 show the evolution of the average of the self-excited vibrations during the transient nonlinear behavior of Case 1, Case 2 and Case 3. As illustrated, the emergence of the new fundamental frequency $f_{1}$ (with its harmonic components and harmonic combinations $\pm n f_{1} \pm m f_{2}$ ) and the global modification of the non-linear vibration behavior is the consequence of the variation of the average non-linear vibration, which is no longer comparable to the equilibrium point of the non-linear system (previously defined in section Instability of the equilibrium point). This fact clearly demonstrates that transient non-linear behavior and modification of the "non-linear equilibrium point" during self-excited vibration are keys for predicting and identifying the fundamental frequencies that govern both the increase of oscillations and transient and stationary vibrations. It can be observed that the global modification of the emergence of non-linear components with the evolution of the average of the self-excited vibrations can be observed in all cases (i.e. $\mu=0.26$-case $1, \mu=0.3$ and $\mu=0.35$ ) even if emergence of new instability does not appear in the three cases.

Thus it clearly appears that different non-linear behaviors can be obtained for the same friction coefficient ( $\mu=0.26$ ) by only introducing a different initial disturbance around the non-linear equilibrium point. As explained previously, in both cases (Case 1 and Case 3), the onset of the non-linear transient vibrations is similar to the emergence of only the fundamental frequency $f_{2}$ and its second harmonic component $2 f_{2}$. Hence the emergence of the new instability of fundamental frequency $f_{1}$ is due only to the "history" of the increase in the self-excited vibrations and the evolutions of contact and loss of contact interactions at the frictional interface. The number of loss of contact is indicated in Figure 10. The phenomenon of contact and loss of contact is only observed during the transient responses for $t=[7 ; 8] s$ with the appearance of the new unstable mode and the "high"" evolution of the average transient vibration. We remind that the stability analysis is not able to predict all the instabilities for this last case the due to the evolution of the average responses (even if no local detachment between the pad and the disc is observed for the stationary responses).

It can be concluded that considering only a stability analysis is not sufficient to predict the full contribution of fundamental frequencies: an under-estimation of the number of unstable modes observed in the nonlinear time simulation can be predicted by the stability analysis. The determination of a non-linear equilibrium point and its stability can only be used as the first step in a global non-linear analysis (i.e. squeal starts under linear conditions around an initial equilibrium point). It is then necessary to calculate the non-linear self-excited vibrations in order to define all the fundamental frequencies (i.e. unstable modes governing the non-linear behavior of the brake system) and the associated harmonics or harmonic combinations. 
(a)

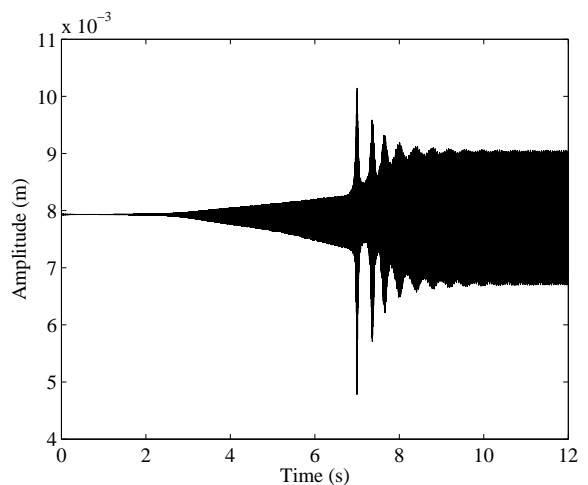

(b)

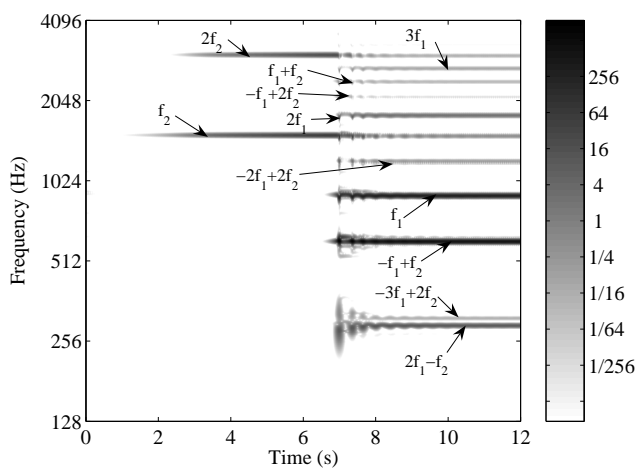

Figure 9: Transient non-linear responses of the brake system for $\mu=0.26$ (a) Time history for $t=[0 ; 12] s$ (b) Wavelet power spectrum for $t=[0 ; 12] s$
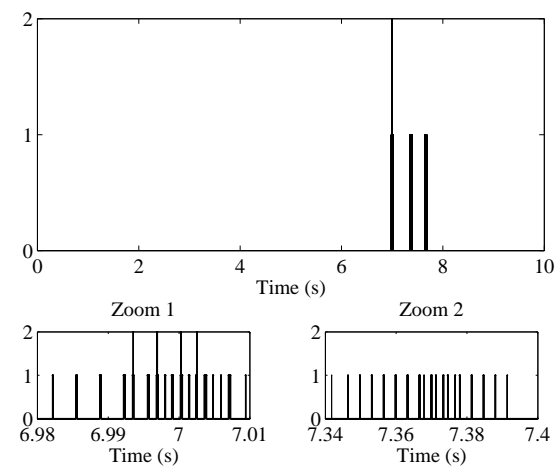

Figure 10: Number of loss of contact during the transient vibration for $\mu=0.26$

\section{Conclusion}

A non-linear model of a disc brake system was developed to study transient and stationary non-linear selfexcited vibrations. The Continuous Wavelet Transform is used to determine the different fundamental frequencies and harmonic combinations of the non-stationary amplitudes of the system. It is demonstrated that when two instabilities occur, the resonance peaks during the transient and stationary vibrations are composed of not only fundamental frequencies $f_{1}$ and $f_{2}$ but also of harmonic components $n f_{i}$ (for $i=1,2$ and $n$ positive integer) and combinations of frequency components $\pm n f_{1} \pm m f_{2}$ (with $n$ and $m$ positive integers). It is observed that the contributions of the harmonic components and the combination of frequency components are essential and cannot be neglected when attemting to avoid poor design. It is also shown that the fundamental frequencies and the lower orders of the harmonic components and harmonic combinations of frequency are generally predominant during transient and stationary vibrations. When only one instability occurs, it is shown that the fundamental frequency and its harmonics can occur during transient and stationary self-excited vibrations. However, it is also illustrated that new fundamental frequencies can appear in the signals, and so non-linear transient amplitudes can become more complex with new contributions due to the coexistence of two instabilities of fundamental frequencies. These results illustrate that the stability analysis of an equilibrium point (classically used as the first step for friction-induced vibration problem) only gives information about the 
(a)

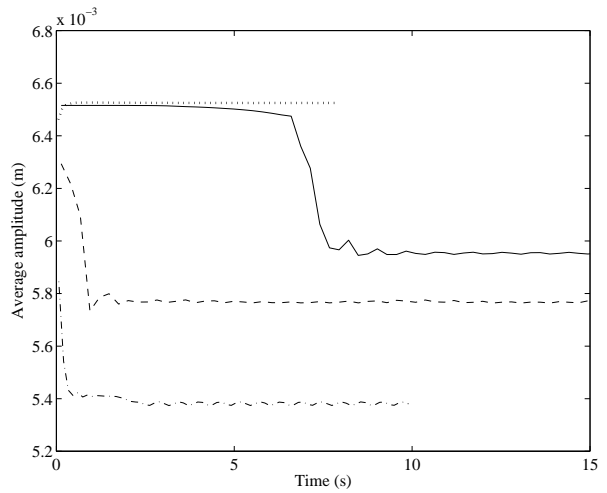

(b)

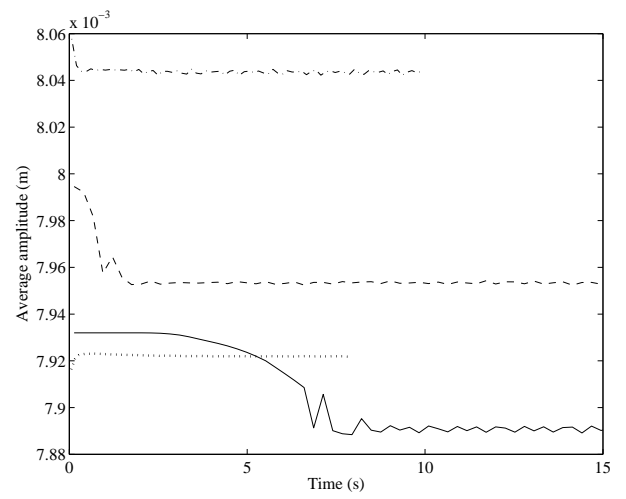

Figure 11: Evolution of the average vibration during test (a) X-direction (b) Y-direction (... $\mu=$ 0.26 for case $1 ;-\mu=0.26$ for case $3 ;--\mu=0.3 ;-.-\mu=0.35$ )

initial rate of increase of disc brake amplitudes. It was demonstrated that an under-estimation of the unstable modes observed in the nonlinear time simulation can be predicted by the stability analysis. During transient vibrations, new fundamental frequencies can be added in the signals due to the contributions of nonlinearities (i.e the nonlinear contact characteristics in the present study) and so the non-linear transient and/or stationary amplitudes can become more complex. Finally it is shown that the transient non-linear amplitudes can be greater than those of the stationary oscillations. Therefore the study of the transient-state behavior has to be taken into account to predict the dynamical response for robust brake system design.

Finally, it is well-known that automobile brakes do not squeal persistently. Generally, self-excited vibrations can occur briefly or intermittently. Thus these facts clearly illustrate that the present study cannot reproduce "real-world situations" exactly. Although extensive studies have been performed in the past decade, many questions in the field of friction-induced vibration remain unanswered and future research is required to increase the reliability and safety of complex automotive brakes. It is not possible to give an exhaustive list of topics of interest for future developments though one of the most crucial steps for future studies is to propose more practical non-linear models capable of considering more realistic physical contributions at friction interfaces. That is to say that variations of friction coefficients and evolutions of nonlinear contact stiffnesses, roughnesses, and contact and loss of contact configurations at the friction interface are essential for studying non-linear selfexcited vibrations (even if all these aspects have been considered in the present study). These variations are capable of modifying the stability of limit cycles and new periodic and non-periodic behaviors can appear.

\section{Acknowledgments}

The author would like to thank PSA (La Garenne-Colombes, Paris, France) for its permission to publish this work. The author would also like to thank the financial support provided by the French National Research Agency through in the framework of its Young Researcher program ANR-07-JCJC-0059-01-CSD 2.

\section{References}

[1] Kinkaid, N., O'Reilly, O., and Papadopoulos, P., 2003. "Automotive disc brake squeal”. Journal of Sound and Vibration, 267, pp. 105-166. 
[2] Ouyang, H., Nack, W., Yuan, Y., and Chen, F., 2005. "Numerical analysis of automotive disc brake squeal : a review". International Journal of Vehicle Noise, 1(3-4), pp. 207-231.

[3] Fritz, G., Sinou, J.-J., Duffal, J.-M., and Jezequel, L., 2007. "Investigation of the relationship between damping and mode-coupling patterns in case of brake squeal". Journal of Sound and Vibration, 307, p. 591-609.

[4] Ouyang, H., and AbuBakar, A., 2006. "Complex eigenvalue analysis and dynamic transient analysis in predicting disc brake squeal". International of Vehicle Noise and Vibration, 2 (2), pp. 143-155.

[5] Massi, F., Baillet, L., Giannini, O., and Sestieri, A., 2007. "Brake squeal: Linear and nonlinear numerical approaches". Mechanical Systems and Signal Processing, 21, p. 2374-2393.

[6] Chen, G. X., Zhou, Z. R., 2007. "Time-frequency analysis of friction-induced vibration under reciprocating sliding conditions". Wear, 262, p. 1-10.

[7] Lorang, X., Foy-Margiocchi, F., Nguyen, Q., and Gautier, P., 2006. “Tgv disc brake squeal”. Journal of Sound and Vibration, 293, p. 735-746.

[8] Kang, J., Krousgrill, C.M., Sadeghi, F., 2009. "Comprehensive stability analysis of disc brake: gyroscopic, negative slope and mode-coupling instability". Journal of Sound and Vibration, 324, p. 387-407.

[9] Kang, J., 2009. "Squeal analysis of gyroscopic disc brake system based on finite element method". International Journal of Mechanical Science, 51, p. 284-294.

[10] Kang, J., Krousgrill, C.M., Sadeghi, F., 2009. "Wave pattern motion and stick-slip limit cycle oscillation of a disc brake". Journal of Sound and Vibration, 325, p. 387-407. Wave pattern motion and stick-slip limit cycle oscillation of a disc brake, JSV 325 (2009)552-564

[11] Coudeyras, N., Sinou, J.-J., and Nacivet, S., 2009. "A new treatment for predicting the self-excited vibrations of nonlinear systems with frictional interfaces: The constrained harmonic balance method, with application to disc brake squeal". Journal of Sound and Vibration, 319(3-5), pp. 1175-1199.

[12] Morlet, J., 1983. "Sampling theory and wave propagation". NATO ASI Series, Springer-Verlag, Berlin, F1, p. 233-261.

[13] Newland, D., 1994. "Wavelet analysis of vibration. part 1: theory". Journal of Vibration and Acoustic, 116, p. 409- 416.

[14] Newland, D., 1993. "An introduction to random vibrations, spectral and wavelet analysis". 3rd ed. Harlow: Longman Scientific and Technical.

[15] Mallat, S., 1989. "Theory for multiresolution signal decomposition: the wavelet representation". IEEE Transaction on Pattern Analysis and Machine Intelligence, 11, p. 674-693.

[16] Chui, C.K., 1992. "An introduction to Wavelets". Academic Press, New York.

[17] Torrence, C., and Compo, G., 1998. "A practical guide to wavelet analysis". Bulletin of the American Meteorological Society, 79(1), pp. 61-78.

[18] Sinou, J.-J., Dereure, O., Mazet, G.-B., Thouverez, F., and Jezequel, L., 2006. "Friction induced vibration for an aircraft brake system. part 1: Experimental approach and stability analysis". International Journal of Mechanical Sciences, 48, pp. 536-554. 